\title{
REDUCING FLOW SEPARATION IN JET PUMPS
}

\author{
M.A.G. Timmer ${ }^{1}{ }^{*}$, J.P. Oosterhuis ${ }^{1}$, S. Bühler ${ }^{1}$, D. Wilcox ${ }^{2}$ and T.H. van der Meer ${ }^{1}$ \\ ${ }^{1}$ Thermal Engineering, Engineering Technology, University of Twente, P.O. Box 217, 7500AE, Enschede, The Netherlands. \\ *Corresponding author's e-mail: timmer.mag@gmail.com \\ ${ }^{2}$ Chart Inc., Troy, New York, USA.
}

Keywords: jet pump, flow separation, acoustic streaming, minor losses

\section{Introduction}

Jet pumps are static components that can be used in closed-loop, traveling wave thermoacoustic devices to suppress a time-averaged mass flux (Gedeon streaming) that can exist [2]. The minimization of convective heat transport caused by this mass flux is of vital importance due to the detrimental effect it has on the device's efficiency. Jet pumps have an asymmetric shape, resulting in asymmetric minor losses. This causes a time-averaged pressure drop that suppresses Gedeon streaming when the minor losses are tuned correctly [1].

Current jet pump designs are mainly based on a quasi-steady approximation using minor loss coefficients [1]. A recent numerical study on conventional jet pump designs has shown that the quasi-steady approximation is only accurate in a small range of operating conditions [4]. It is shown that, above certain wave amplitudes, flow separation occurs during the half-cycle where the bulk flow is moving in the diverging direction of the jet pump. The flow separation originates from the small jet pump opening as a result of the local adverse pressure gradient. Due to the flow separation the asymmetry in minor losses diminishes, resulting in a severe downgrade of the jet pump performance compared with the quasi-steady approximation. The current work will investigate the flow separation behavior by varying the geometry of the jet pump. The goal is to minimize the flow separation and shift it to higher wave amplitudes, therewith increasing the effectiveness and robustness of jet pumps.

\section{Method}

In a similar manner as in Oosterhuis [4], an axisymmetric computational fluid dynamics model is developed which solves the unsteady, fully compressible Navier-Stokes equations. The boundary conditions enforce a $20 \mathrm{~Hz}$ traveling wave, with a wave amplitude that maintains flow in the laminar regime according to Ohmi and Iguchi [3].

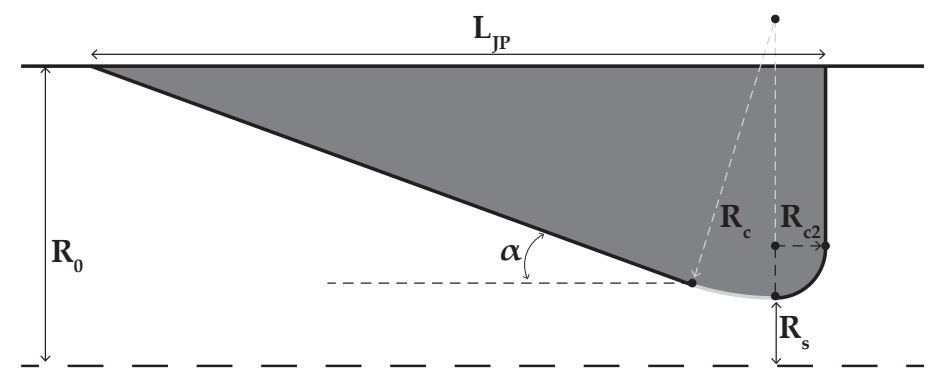

Figure 1: Axisymmetric representation of the jet pump geometry. Bottom dashed line depicts the symmetry axis, top solid line indicates the outer tube wall.

The investigated jet pump geometry is depicted in Figure 1. Conventional jet pumps use a single radius of curvature to reduce the minor loss coefficient at the small opening $\left(R_{c}=R_{c 2}\right)$, with the corresponding jet pump length defined as the reference length $\mathrm{L}_{\mathrm{JP}}$,ref. In an effort to minimize and shift flow separation, the adverse pressure gradient is reduced by increasing 
the radius of curvature $R_{c}$. This introduces a longer and smoother transition from the small opening to the tapered surface. The extra jet pump length introduced by this longer transition with respect to $\mathrm{L}_{\mathrm{JP} \text {,ref }}$ is defined as $\Delta \mathrm{L}_{\mathrm{JP}}$. Six cases are compared with the reference, namely: $\Delta \mathrm{L}_{\mathrm{JP}}=3,5,10,20,30$ and $50 \mathrm{~mm}$. The other dimensions are kept constant and are given by: $\mathrm{R}_{0}=30 \mathrm{~mm}, \mathrm{R}_{\mathrm{s}}=7 \mathrm{~mm}, \mathrm{R}_{\mathrm{c} 2}=5 \mathrm{~mm}, \alpha=15^{\circ}$ and $\mathrm{L}_{\mathrm{JP}, \mathrm{ref}}=91.5 \mathrm{~mm}$ (for $\mathrm{R}_{\mathrm{c}}=5 \mathrm{~mm}$ ).

\section{Results and discussion}

The jet pump performance is studied in terms of the dimensionless time-averaged pressure drop $\Delta p_{2}^{*}$ and acoustic power dissipation $\Delta \dot{E}_{2}^{*}$ [4], which are depicted in Figure 2 as a function of the Keulegan-Carpenter number. The latter is a dimensionless measure of the wave amplitude scaled with the jet pump waist diameter $\left(2 R_{s}\right)$. For larger transition lengths, the maximum pressure drop increases significantly. Additionally, the point at which the pressure drop is at its maximum shifts to higher amplitudes. A flow field investigation shows that this is the result of flow separation being shifted to higher $K C_{D}$ values. Before flow separation occurs, the pressure drop of the cases with the largest transition lengths is similar to the quasi-steady approximation. The acoustic power dissipation is reduced for larger transition lengths.
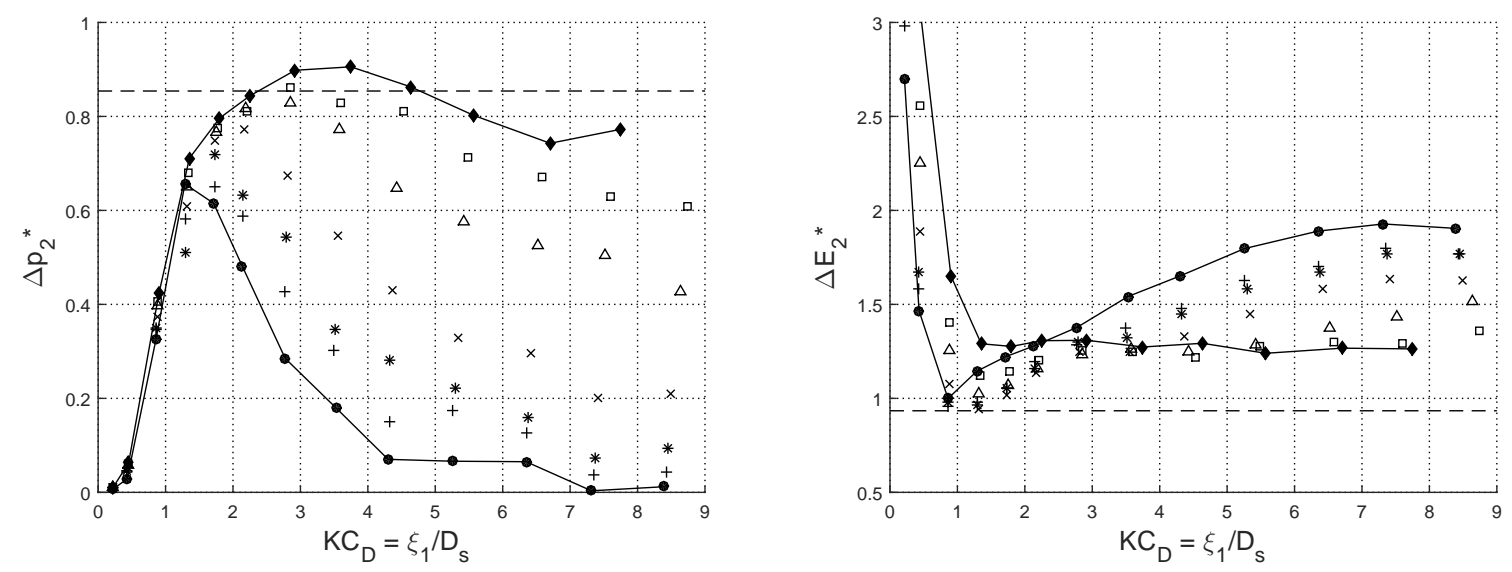

Figure 2: Dimensionless pressure drop (left) and dimensionless acoustic power dissipation (right) for all cases, with: $\Delta L_{J P}=0 \mathrm{~mm}(\bullet$, reference $), 3 \mathrm{~mm}(+), 5 \mathrm{~mm}(*), 10 \mathrm{~mm}(\times), 20 \mathrm{~mm}(\triangle), 30 \mathrm{~mm}(\square)$ and $50 \mathrm{~mm}(\bullet)$. The dashed lines represent the quasi-steady approximation and the solid lines the cases with the smallest and largest transition length.

\section{Conclusions and outlook}

By prolonging the transition length between the small opening and the tapered surface of jet pumps, flow separation is shifted to higher amplitudes and the pressure drop is increased significantly. This results in an improvement of the effectiveness and robustness of jet pumps. Future work will focus on reducing the jet pump length without downgrading its performance.

\section{Acknowledgements}

Bosch Thermotechnology and Agentschap NL are thankfully acknowledged for the financial support as part of the EOS-KTO research program under project number KTOT03009.

\section{References}

[1] Backhaus, S. and Swift, G. "A thermoacoustic-Stirling heat engine: detailed study". J. Acoust. Soc. Am., 107, (2000), 3148-3166.

[2] Gedeon, D. “DC Gas Flows in Stirling and Pulse Tube Cryocoolers". In: “Cryocoolers 9", (1997), 385-392.

[3] Ohmi, M. and Iguchi, M. “Critical Reynolds number in an oscillating pipe flow”. B. JSME, 25, (1982), $165-172$.

[4] Oosterhuis, J. P., Bühler, S., van der Meer, T. H., and Wilcox, D. "A numerical investigation on the vortex formation and flow separation of the oscillatory flow in jet pumps". J. Acoust. Soc. Am., 137, (2015), 1722-1731. 